Research Article

Open Access

\title{
Comparative study of Four Layer Compression Bandaging and Topical Human Epidermal Growth Factor in Chronic Venous Leg Ulcer
}

\author{
Balasubrahmanya KS, Vinay $\mathbf{G}^{\star}$, Srinidhi M and Sunil Kumar APV \\ Department of General Surgery, Mysore Medical College and Research Institute, Mysore, Karnataka, India
}

\section{Article Info}

*Corresponding author: Vinay $\mathbf{G}$

Department of General Surgery

Mysore Medical College and Research

Institute

Mysore, Karnataka,

India

Tel: +91 9986929929

E-mail: dr.vinay1990@gmail.com

Received: January 25, 2018

Accepted: February 17, 2018

Published: February 23, 2018

Citation: Balasubrahmanya KS, Vinay G, Srinidhi M, Sunil Kumar APV. Comparative study of Four Layer Compression Bandaging and Topical Human Epidermal Growth Factor in Chronic Venous Leg Ulcer. Madridge J Surg. 2018; 1(1): 24-28.

doi: $10.18689 / \mathrm{mjs}-1000106$

Copyright: (c) 2018 The Author(s). This work is licensed under a Creative Commons Attribution 4.0 International License, which permits unrestricted use, distribution, and reproduction in any medium, provided the original work is properly cited.

Published by Madridge Publishers

\begin{abstract}
Background: Leg ulcers are defined to be loss of epidermal and dermal tissues of leg or foot. Approximately $1 \%$ of the population suffer from a leg ulcer at some stage during their lives. Venous leg ulcers are consequence of chronic venous insufficiency.

Aims and Objectives: The aim of the study is to compare the healing rate and area of healing in chronic venous ulcer with the topical use of Human epidermal growth factor (H-EGF) along with regular four layer compression bandaging as versus four layer compression bandaging alone.
\end{abstract}

Materials And Methods: A Prospective, open label, randomized, comparative, hospital based, single centered study was conducted among 90 subjects attending General surgery OPD, K.R. Hospital attached to Mysore Medical College And Research Institute, Mysore meeting the inclusion and exclusion criteria over a period of 24 months from January 2016 to December 2017. All diagnosed cases of chronic venous ulcer were included for the study. Venous ulcers subjects with arterial insufficiency $(A B P I<0.8)$ and all other causes of chronic ulcers such as diabetes, trophic, arterial ulcers were excluded from the study. Descriptive statistics, Chi-square test and Student-t-test were used to analyse the results.

Results: The mean age group of the study subjects was $51.43 \pm 7.436$ years in H-EGF group and $54.86 \pm 10.341$ years in four layered dressing group. The gender distribution showed 86(95.56\%) males as compared to 4(4.44\%) females. Culture shows E.Coli in 7(15.56\%) and $5(11.11 \%)$ subjects in H-EGF group and four layer dressing group respectively. 3(6.67\%) and $1(2.22 \%)$ subjects had there culture positive for streptococcus in H-EGF group and four layer dressing group respectively. The H-EGF dressing group was very effective as there was faster ulcer healing when compared to regular four layered dressing.

Conclusion: Topical H-EGF along with four layer dressing can be used in chronic venous leg ulcer, as it significantly hastens healing time than the conventional four layer bandaging technique alone.

Keywords: Four layer compression bandaging; Human epidermal growth factor; Chronic venous leg ulcer Healing.

\section{Introduction}

Leg ulcers are defined to be loss of epidermal and dermal tissues of leg or foot. Leg ulcers are most commonly caused due to venous Insufficiency, arterial insufficiency, neuropathy often due to diabetes and ulcer from prolonged pressure and ischemia [1]. Less common causes include; trauma, inflammation, malignancy, metabolic Conditions and due to 
Infections. Approximately $1 \%$ of the population suffers from a leg ulcer at some stage during their lives [2]. Venous leg ulcers are consequence of chronic venous insufficiency [3].

The goals of non-operative treatment: for venous ulceration are to promote healing of the existing ulcer and prevent recurrences while allowing the patient to maintain a normal ambulatory status. Compression therapy remains the primary non-operative treatment for chronic venous insufficiency despite progress in both ablative and reconstructive venous surgery [4].

Compression $=\mathrm{N}$ (No. of Bandage Layer) $x \mathrm{t}$ (Bandage Tension) $\mathrm{R}$ (Radius of Leg)

Compression pressure of a least $30 \mathrm{~mm} \mathrm{Hg}$ to $40 \mathrm{~mm}$ of $\mathrm{Hg}$ at the ankle should be utilized in the management of venous leg ulcers. All compression bandage systems must create a pressure gradient from ankle to knee. According to the law of laplace, which mathematically relates bandage tension compression pressure, the shape of leg will create this gradient. Hence compression will be found maximum at the gaiter area just proximal to ankle joint of $30-42 \mathrm{~mm} \mathrm{Hg}$ and as we go up the leg it decreases and at the knee it in 17$20 \mathrm{~mm} \mathrm{Hg}$ [5].

Recommended pressures for the treatment of venous disorder include; ankle pressure of $14-17 \mathrm{~mm} / \mathrm{Hg}$ in superficial or early varices, $18-24 \mathrm{~mm} / \mathrm{Hg}$ in varices of medium severity or with ulcer treatment and prevention of mild edema and $25-35 \mathrm{~mm} / \mathrm{Hg}$ in gross varices, post-thrombotic syndrome, gross edema, ulcer treatment and prevention [5].

First layer dressing - Gamgee applied from foot to above knee to absorb the discharge from ulcers and protect bony prominences.

Second layer - Banding with cotton bandage to keep the 1 st layer in place

Thirdlayer - Crepe bandage which applies the compression pressure.

Four layer - Micropore to keep compression bandage in place.

Type of bandages $[6,7,8]$ :

Type 1 - Light weight conforming - stretch bandages. Which have simple dressing retention function. They have light weight elastomeric threads, which impart a high degree of elasticity but little power to the bandage.

Type 2 - Light support bandages called short or minimal stretch bandages. The crepe -type products of British pharmacopoeia. These have been used for the treatment of venous ulcer. They form an inelastic covering to the leg which tends to resist any change in the geometry of the calf muscle during exercise.

Type 3 - Compression bandages, deliberate application of pressure to control edema and reduce swelling.

Type $3 a$ - Light compression bandages maintain low level of pressure up to $20 \mathrm{~mm} \mathrm{Hg}$ indicated in superficial or early varices and varicosis during pregnancy.
Type 3b - Moderate compression bandages, pressure of 30 $\mathrm{mmHg}$ the ankle, used in the treatment of varicosis during pregnancy, varices of medium severity.

Type $3 c$ - Have pressure of $40 \mathrm{~mm}$ of $\mathrm{Hg}$ - Gross varices, gross edema, post thombotic venous insufficiency and leg ulcers.

Type 3d - extra high performance compression bandages have pressure of $>50 \mathrm{mmHg}$ used for most edematous limbs.

Recombinant human EGF produced in E.Coli is a single, non-glycosylated polypeptide chain containing 53 amino acids and having a molecular mass of 6222 dalton. The r-HuEGF is purified by proprietary chromatographic techniques. Escherichia Coli is the source. The sequence of the first five $\mathrm{N}$-terminal amino acids was determined and was found to be Asn-Ser-Asp-Ser-Glu, which agrees with the sequence of native human EGF. N- Terminal methionine has been completely removed enzymatically. Dimmers and aggregates less than $1 \%$ as determined by silver-stained SDS-PAGE gel analysis.

Lyophilized r-Hu-EGF although stable at room temperature for 3 weeks, should be stored desiccated below -18 degree celsius. Upon reconstitution r-Hu-EGF should be stored at 4 degree celsius. between 2-7 days and for future use below -18 degree celsius. For long term storage it is recommended to add a carrier protein $(0.1 \%$ HAS or BSA). Please prevent freeze-thaw cycles.

Recombinant human EGF is fully biologically active when compared to standards. The ED50, calculated by the dosedependent proliferation of murine BALB/c 3T3 cells (measured by $3 \mathrm{H}$-thymidine uptake) is $<0.1 \mathrm{ng} / \mathrm{ml}$ corresponding to a specific activity of $1 \times 10^{7}$ units / mg.

Protein quantitation was carried out by UV spectroscopy at $280 \mathrm{~nm}$ using the absorbency value of 2.858 as the extinction coefficient for a $0.1 \%$ solution. This value is calculated by the PC GENE computer analysis program of protein sequences (intelli Genetics) and analysis by RP-HPLC, using a calibrated solution of EGF as a reference standard [9, 10].

The aim of the study is to compare the healing rate and area of healing in chronic venous ulcer with the topical use of $\mathrm{H}$-EGF along with regular four layer compression bandaging as versus four layer compression bandaging alone.

\section{Materials and Methods}

A prospective, open label, randomized, comparative, hospital based, single centered study was conducted among 90 subjects attending General Surgery OPD, K.R. Hospital attached to Mysore Medical College And Research Institute, Mysore meeting the inclusion and exclusion criteria over a period of 24 months from January 2016 to December 2017.

Patients aged more than 18 years with clean chronic venous ulcers of lower extremity were included in the study. Venous ulcers patients with arterial insufficiency ( $A B P I<0.8$ ) and all other causes of chronic ulcers such as diabetes, trophic, arterial ulcers were excluded from the study. 
All patients who presented to the hospital with complaints of non healing varicose ulcer were subjected to a detailed history and clinical examination. Size of ulcer was measured by planimetry with a sterile guaze or graph paper. Patients were randomly put into test and control group. Relevant investigation done like $\mathrm{Hb} \%$, RBS, Doppler venous scan. Patient in H-EGF group were subjected to four layer dressings with human epidermal growth factor topically applied to the ulcer and other group of patients were subjected to regular four layer dressing, every week. Patients were followed up every week until ulcer healed completely.

Descriptive statistics, Chi-square test and Student-t-test were used to analyse the results.

\section{Results}

During the 24 months study period, 45 patients had their wounds treated by human epidermal growth factor (H-EGF) gel and 45 patients underwent four layer dressings. The mean age group of the study subjects was $51.43 \pm 7.436$ (32-76) years in H-EGF group and $54.86 \pm 10.341$ (38-81) years in four layered dressing group. The gender distribution showed 86(95.56\%) males as compared to $4(4.44 \%)$ females. $16(35.56 \%)$ subjects were diabetic and $12(26.67 \%)$ subjects were hypertensive in H-EGF group and 14(31.11\%) subjects were diabetic and 9(20\%) subjects were hypertensive in four layer dressing group.

Culture shows E.Coli in 7(15.56\%) and 5(11.11\%) subjects in H-EGF group and four layer dressing group respectively. 3(6.67\%) and $1(2.22 \%)$ subjects had their culture positive for streptococcus in H-EGF group and four layer dressing group respectively. Incompetence of Venous segments in H-EGF group is 29(64.44\%) and 26(57.78\%) in four layer dressing group had SF incompetence and; $8(17.78 \%)$ and no subjects had SPJ incompetence in four layer dressing group and H-EGF group respectively. $36(80 \%)$ in H-EGF group and $31(68.89 \%)$ in four layer dressing group had incompetence of perforators. $35(77.78 \%)$ and $28(62.22 \%)$ had ulcer in the left leg in H-EGF group and four layer dressing group respectively. 10(22.22\%) in H-EGF group and $17(37.78 \%$ ) in four layer dressing group had ulcer in the right leg. 30(66.67\%) and $27(60 \%)$ subjects had ulcer for $6-12$ months duration in H-EGF group and four layer dressing group respectively.

12(26.67\%), 26(57.78\%) and 7(15.56\%) subjects in H-EGF group and $8(17.78 \%), 24(53.33 \%)$ and $13(28.89 \%)$ subjects in four layer dressing group had $<3,3-6$ and $>6 \mathrm{cms}$ ulcer respectively.

According to the gender; the mean healing duration was 5.06 weeks in males and 5.23 weeks in females among $\mathrm{H}$-EGF group and 6.57 weeks in males and 7.29 weeks in females among four layer dressing group (Table 1, Graph 1).

Table 1. Comparison of Mean Healing Duration according to Gender in H-EGF and Four Layer Dressing Group $(n=90)$

\begin{tabular}{|c|c|c|c|c|c|c|}
\hline Group & Gender & $\mathrm{N}$ & $\begin{array}{c}\text { Mean Follow Up } \\
\text { (Weeks) }\end{array}$ & $\begin{array}{l}\text { Standard } \\
\text { Deviation }\end{array}$ & t-Value & $p$-Value \\
\hline \multirow{2}{*}{ H-EGF Group } & Male & 42 & 5.06 & 1.658 & \multirow{2}{*}{0.006} & \multirow{2}{*}{0.957} \\
\hline & Female & 3 & 5.23 & 1.834 & & \\
\hline \multirow{2}{*}{$\begin{array}{c}\text { Four Layer } \\
\text { Dressing Group }\end{array}$} & Male & 44 & 6.57 & 2.043 & \multirow{2}{*}{1.247} & \multirow{2}{*}{0.235} \\
\hline & Female & 1 & 7.29 & 1.787 & & \\
\hline
\end{tabular}

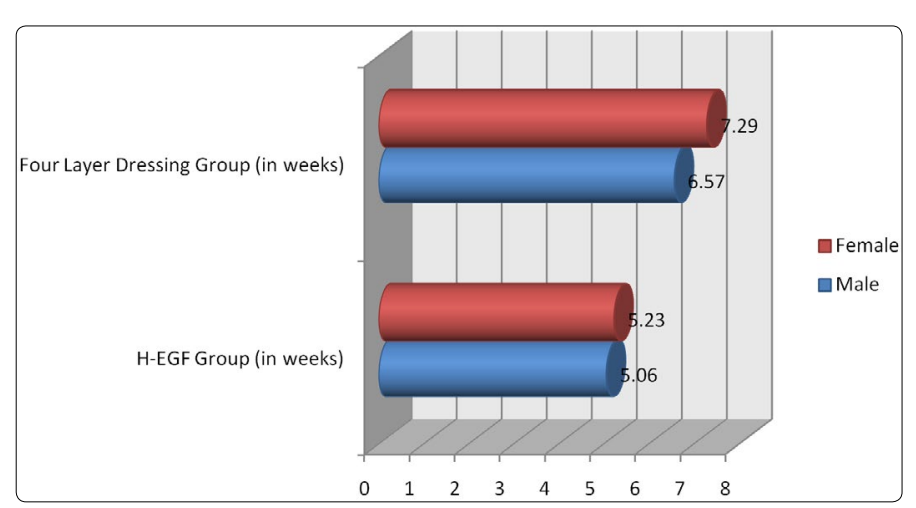

Graph 1. Comparison of Mean Healing Duration according to Gender in H-EGF and Four Layer Dressing Group $(n=90)$

According to the incompetence of venous segments involved; the mean healing duration in H-EGF group is 4.36 weeks and 6.41 weeks in four layer dressing group for patients with SFJ incompetence. 0 weeks in H-EGF group and 7.27 weeks in four layer dressing group for patients with SPJ incompetence. 4.89 weeks in H-EGF group and 7.66 weeks in four layer dressing group for patients with perforator incompetence (Table 2, Graph 2).

Table 2. Comparison of Mean Healing Duration According to Incompetence of Venous Segments in H-EGF and Four Layer Dressing Group ( $n=90)$

\begin{tabular}{|c|c|c|c|c|c|c|}
\hline & Group & $\mathrm{N}$ & $\begin{array}{c}\text { Mean } \\
\text { Follow Up } \\
\text { (Weeks) }\end{array}$ & $\begin{array}{l}\text { Standard } \\
\text { Deviation }\end{array}$ & t-Value & p-Value \\
\hline \multirow{2}{*}{$\begin{array}{c}\text { Sapheno } \\
\text { Femoral } \\
\text { Junction } \\
\text { (SFJ) }\end{array}$} & H-EGF Group & 29 & 4.36 & 2.651 & \multirow[b]{2}{*}{1.264} & \multirow[b]{2}{*}{0.674} \\
\hline & $\begin{array}{c}\text { Four Layer } \\
\text { Dressing Group }\end{array}$ & 26 & 6.41 & 1.967 & & \\
\hline \multirow{2}{*}{$\begin{array}{c}\text { Sapheno } \\
\text { Popliteal } \\
\text { Junction (SPJ) }\end{array}$} & H-EGF Group & 0 & 0 & - & \multirow[b]{2}{*}{ - } & \multirow[b]{2}{*}{-} \\
\hline & $\begin{array}{c}\text { Four Layer } \\
\text { Dressing Group }\end{array}$ & 8 & 7.27 & 2.235 & & \\
\hline \multirow{2}{*}{$\begin{array}{c}\text { Perforator } \\
\text { Incompetence }\end{array}$} & H-EGF Group & 36 & 4.89 & 3.005 & \multirow[b]{2}{*}{0.843} & \multirow[b]{2}{*}{0.231} \\
\hline & $\begin{array}{c}\text { Four Layer } \\
\text { Dressing Group }\end{array}$ & 31 & 7.66 & 2.659 & & \\
\hline
\end{tabular}

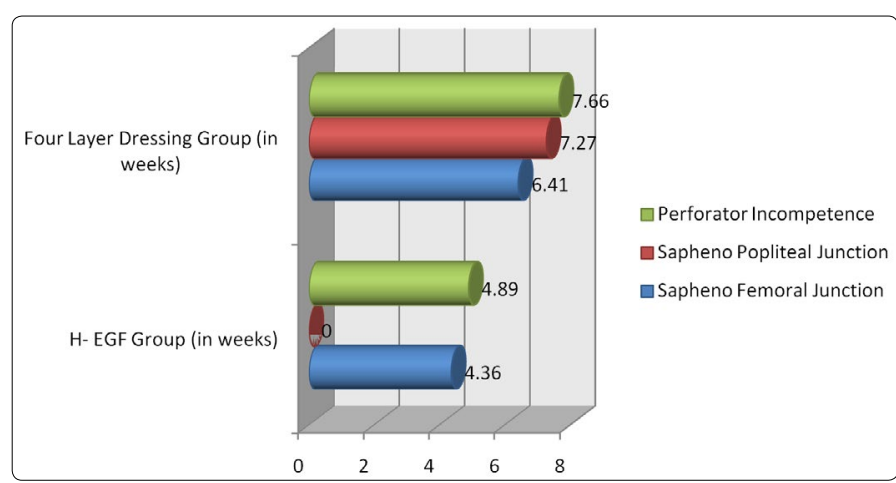

Graph 2. Comparison of Mean Healing Duration According to Incompetence of Venous Segments In H-EGF and Four Layer Dressing Group ( $\mathrm{n}=90)$

According to ulcer size; the mean healing time in H-EGF group was 3.44 weeks and in four layer dressing group was 5.62 weeks for ulcer $<3 \mathrm{cms}$ ( $p 0.0032$ ). The mean healing time in H-EGF group was 4.37 weeks and in four layer dressing group was 6.21 weeks for ulcer $3-6 \mathrm{cms}$ ( $p$ 0.0014). The mean healing time in H-EGF group was 6.73 weeks and in four layer dressing group was 7.86 weeks for ulcer $>6 \mathrm{cms}$ ( $p$ 0.0045). The mean standard deviation was $5.98 \pm 1.664$ in H-EGF group and $7.35 \pm 2.173$ in four layer dressing group (Table 3, Graph 3). 
Table 3. Comparison of Mean Healing Duration According to Ulcer Size In H-EGF and Four Layer Dressing Group $(n=90)$

\begin{tabular}{|c|c|c|c|c|c|c|}
\hline $\begin{array}{l}\text { Ulcer } \\
\text { Size } \\
(\mathrm{cms})\end{array}$ & Group & $\mathrm{N}$ & $\begin{array}{c}\text { Mean } \\
\text { Follow Up } \\
\text { (Weeks) }\end{array}$ & $\begin{array}{l}\text { Standard } \\
\text { Deviation }\end{array}$ & t-Value & $p$-Value \\
\hline \multirow[b]{2}{*}{$<3 \mathrm{cms}$} & H-EGF Group & 12 & 3.44 & 1.087 & \multirow[b]{2}{*}{14.386} & \multirow[b]{2}{*}{0.0032} \\
\hline & $\begin{array}{c}\text { Four Layer } \\
\text { Dressing Group }\end{array}$ & 8 & 5.62 & 0.892 & & \\
\hline \multirow[b]{2}{*}{$3-6 \mathrm{cms}$} & H-EGF Group & 26 & 4.37 & 1.265 & \multirow[b]{2}{*}{40.558} & \multirow[b]{2}{*}{0.0014} \\
\hline & $\begin{array}{c}\text { Four Layer } \\
\text { Dressing Group }\end{array}$ & 24 & 6.21 & 1.054 & & \\
\hline \multirow[b]{2}{*}{$>6 \mathrm{cms}$} & H-EGF Group & 7 & 6.73 & 1.371 & \multirow[b]{2}{*}{12.492} & \multirow[b]{2}{*}{0.0045} \\
\hline & $\begin{array}{c}\text { Four Layer } \\
\text { Dressing Group }\end{array}$ & 13 & 7.86 & 1.078 & & \\
\hline \multirow[b]{2}{*}{ Total } & H-EGF Group & 45 & 5.98 & 1.664 & \multirow[b]{2}{*}{24.566} & \multirow[b]{2}{*}{$<0.001$} \\
\hline & $\begin{array}{c}\text { Four Layer } \\
\text { Dressing Group }\end{array}$ & 45 & 7.35 & 2.173 & & \\
\hline
\end{tabular}

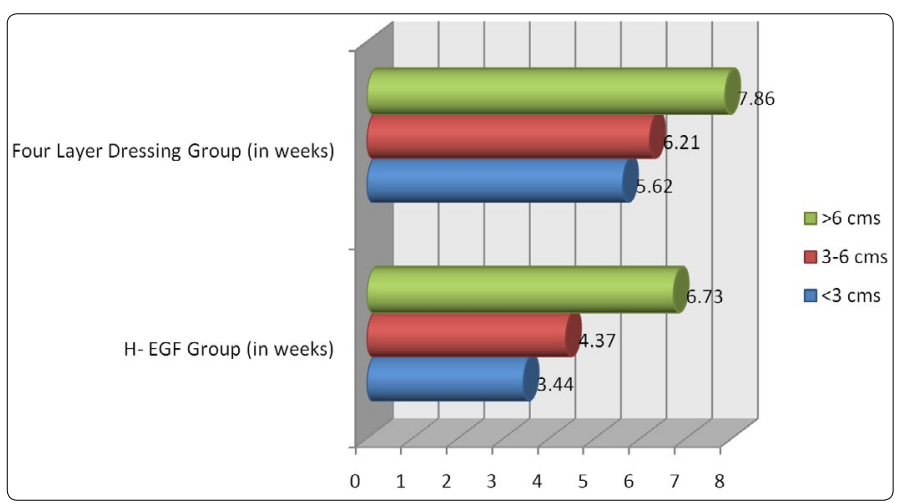

Graph 3. Comparison of Mean Healing Duration According to Ulcer Size in H-EGF and Four Layer Dressing Group $(n=90)$

\section{Discussion}

The intrinsic biological and physico-chemical characteristics of human epidermal growth factor, have been exploited to prepare a variety of commercially available medical products. A great deal of interest has been focused recently on the potential use of synthetic polypeptide growth factor to stimulate healing of chronic wound.

Falanga.V.Eaglstein WH et al [11], in their pilot doubleblind randomized study, conducted at a single center; used $\mathrm{H}$-EGF to treat 44 patients with venous ulceration of lower extremities, 22 EGF, 22 placebo. They concluded - 35patients (17-H-EGF-18placebo - 9 excluded) were evaluated. Follow up period was 10 weeks, median base line ulcer size -18.5 $\mathrm{cm}^{2}$, H-EGF group - $12.9 \mathrm{~cm}^{2}$, Placebo group - $19.2 \mathrm{~cm}^{2}$ $(p=.27)$ (no significant difference). 6 (35\% - H-EGF group), 2 (11\% - placebo group healed completely $(p=10) .6$ (2 of 17 H-EGF group, 4 of 18 Placebo group developed healthy granulations suitable for grafting $(p=.50)$.

- Median ulcer size reduction was - 7\% for H-EGF group, $3 \%$ for placebo group per week, $p$ value $=0.29$.

- Overall $73 \%$ versus $33 \%$ at study end $p=0.32$.

- No untoward side effects were related to the application of H-EGF group.

- Concluded that topical application of H-EGF group in the dose and the manner used was safe in this study. There was greater reduction in ulcer size.

- A large no of healed ulcers with the of H-EGF group are encouraging results.
F.J.Meyer et al [12], in their prospective randomized study of 133 patients, compared four layer dressing with 3 layer paste bandages in the treatment of venous leg ulcers. Result were $51-80 \%$ of 64 in 3 layer paste bandages ulcer healed completely, $45-65 \%$ of 69 patients with four layer healed completely ( $p=0.031), 12$ weeks median time - for three layer group and 16 weeks for - four layer group $(p=0.040)$. They Concluded three layer paste bandages were significantly more effective at healing venous ulcers than four layer regimen.

Scriven et al [13], did a prospective randomized trial of four layer bandaging versus short stretch compression bandaging for venous leg ulcers. 53 patient included in study, four layer had 32 patients, ssb had 32 patients, 50 completed the protocol, Healing rate $-55 \%$ - for four layer, $57 \%$ - for ssb - chi $2=0.0, \mathrm{df}=1, \mathrm{P}=1.0$ Lager ulcer $>10 \mathrm{~cm}^{2}$ took longer to heal, smaller ulcer then $<10 \mathrm{~cm}^{2}$ lesser time to heal they Concluded that four layer and short stretch bandage were equally efficacious in healing venous ulcers four layer dressing limb had fewer complication than ssb, ulcer healing rates was not influenced by presence of deep venous reflux, post thrombotic deep vein changes nor by ulcer duration.

Nelson, et al [14], showed $67 \%$ healing rates of venous ulcers within 24weeks when using four layer compression therapy and $49 \%$ healing rates with single layer compression therapy.

\section{Conclusion}

The results from present study show that four layer bandaging along with topical application of $\mathrm{H}-\mathrm{EGF}$ in treating varicose ulcers is better than the conventional four layer compression bandaging alone.

H-EGF promotes faster healing by promoting granulation and accelerating the rate of epithelization. There were no side effects observed due to H-EGF.

Therefore it can be positively concluded that topical H-EGF along with four layer dressing can be used in the management of varicose ulcer, as it significantly hastens healing time in varicose ulcers than the conventional four layer bandaging technique alone.

However there were few limitations in our study which include; small sample size, open label, hospital based and single center study. Further studies evaluating this, overcoming the above limitations is highly desired.

\section{Compliance with Ethical Standards}

Informed consents were taken from all participants, and the study protocol was approved by Institutional ethical committee approval board.

\section{Conflict of interest}

The authors confirm that there is no conflict of interest regarding this manuscript. 


\section{References}

1. Brown V, Marshal D. Compression therapy in ulcer management. Nursing Standard. 2001; 15(22)64-70. doi: 10.7748/ns.15.22.64.s60

2. Bello YM, Phillips TJ. Chronic leg ulcers: types and treatment. Hosp Pract. 2000; 128-129.

3. Conrad G, Cliniques GL. Ulceration related to varicose vein insufficiency. Wound Care Institute, Inc. 1998; 561: 624-634.

4. Backhouse CM, Blair SD, Savage AP, Walton J, McCollum CN. Controlled trial of occlusive dressing in healing chronic venous ulcer, $B r J$ Surg. 1987; 74(7): 626-627.

5. Stephen Thomas. Compression bandaging in the treatment of venous leg ulcers, Director Surgical Materials Testing Laboratory. Edition 1.1. 1998.

6. Partsch $\mathrm{H}$, Do we need firm compression stockings exerting high pressure? Vara. 1984; 13(1): 52-57.

7. Gaylarde PM, Sarkany I, Dodd HJ. The effect of compression on venous stasis. Br J Dermatology. 1993; 128(3): 255-258. doi: 10.1111/j.1365-2133.1993. tb00167.x
8. Partsh H. Compression therapy of the legs. A review. J Dermatol Surg Oncol. 1991; 17(10): 799-805.

9. Human Epidermal growth factor, recombinant Sep, 92008.

10. Mroczkowski B, Reich $\mathrm{M}$, Chen $\mathrm{K}$, Bell Gl, Cohen S. Recombinant human epidermal growth factor precursor is a glycosylated membrane protein with biological activity. Mol Cell Biol. 1989; 9(7): 2771-2778.

11. Falanga V, Eaglstein WH, Bucalo B, KatzMH, hersin B, Carson P.Topical use of human Recombinant epidermal growth factor (H-EGF) in venous ulcers. J Dermatol surg oncol. 1992; 18(7); 604-606.

12. Meyer FJ, McGuinness CL, Lagattolla NR, Eastham D, Burnand KG. Randomised clinical trial of three layer paste and four layer bandages for venous leg ulcers. Br J Surg. 2003; 90(8): 934-940. doi: 10.1002/bjs.4173

13. Scriven JM, Taylor LE, Wood AJ, Bell PR, Naylor AR, et al. Prospective randomized trial of four-layer versus short stretch compression bandages for the treatment of venous leg ulcers. Ann R Coll Surg Engl. 1998; 80(3): $215-20$.

14. Nelson EA, Iglesian CP, Cullum N, Torgerson DJ. Randomined clinical trials of four layer and short-stretch compression bandages for venous leg ulcer (Venous-1). Br J Surg. 2004; 91(10): 1292-1299. doi: 10.1002/bjs.4754 